\title{
Skyrme-RPA study of charged-current neutrino opacity in hot and dense su- pernova matter
}

\author{
Alan A. Dzhioev ${ }^{1, *}$ and Gabriel Martínez-Pinedo ${ }^{2,3, * *}$ \\ ${ }^{1}$ Bogoliubov Laboratory of Theoretical Physics, JINR, 141980, Dubna, Russia \\ ${ }^{2}$ GSI Helmholtzzentrum für Schwerionenforschung, Planckstraße 1, 64291 Darmstadt, Germany \\ ${ }^{3}$ Institut für Kernphysik (Theoriezentrum), Technische Universität Darmstadt, Schlossgartenstraße 2, 64289 Darmstadt, Germany
}

\begin{abstract}
Neutrino emission and their transport of energy to the supernova shock region are sensitive to the physics of hot and dense nuclear matter, which is a complex problem due to the strong correlations induced by nuclear forces. We derive charged-current opacities for electron neutrinos and antineutrinos in supernova matter using a self-consistent approach based on the Skyrme effective interaction. We include a mean field energy shift due to nuclear interaction and corrections due to RPA correlations. A complete treatment of the full Skyrme interaction, including a spin-orbit term, is given. To test the effect of RPA corrections, neutrino and antineutrino opacities are computed using different Skyrme parameterisations consistent with a number of infinite matter constraints.
\end{abstract}

\section{Introduction}

Neutrino and antineutrino absorption reactions in nuclear matter play a crucial role in core-collapse supernovae [1, 2]. In delayed neutrino-heating mechanism, the energy transport by electron neutrinos and antineutrinos from a hot protoneutron star (PNS) is responsible for reviving the stalled shock-wave and generating an explosion [3, 4]. The charged-current reactions $v_{e}+n \rightarrow p+e$ and $\bar{v}_{e}+p \rightarrow n+e^{+}$ provide a dominant mechanism for heating the matter behind the shock. The efficiency of neutrino-heating mechanism and the success of the explosion depend critically on the luminosity and energy spectra for $v_{e}$ and $\bar{v}_{e}$. In turn, these characteristics are determined by the neutrino transport inside the protoneutron star, and thus depend on the neutrino opacities in the dense $\left(\rho \simeq 10^{12}-10^{14} \mathrm{~g} / \mathrm{cm}^{3}\right)$ and hot $(T \simeq 5-10 \mathrm{MeV})$ nuclear matter. The main opacity sources for $v_{e}$ and $\bar{v}_{e}$ are the same absorption reactions on nucleons, which are responsible for the neutrino-heating mechanism.

For dense neutron-rich conditions relevant in the protoneutron star, earlier calculations have found that manybody correlations due to strong interactions between nucleons can modify the neutrino opacities significantly. In particular, it was found $[5,6]$ that the difference in the neutron and proton mean-field potential energies enhances (suppresses) the (anti)neutrino opacity considerably with respect to that in the noninteracting gas of free nucleons. Meanwhile, in [6] it was shown that random-phase approximation corrections, accounting for long-range $p h$ correlations, act in the opposite direction, but their effect is less

\footnotetext{
*e-mail: dzhioev@theor.jinr.ru

**e-mail: g.martinez@gsi.de
}

significant as compared to that of mean-field potential difference.

It was emphasized in many works (see, for example, Refs. [7-9]) that neutrino opacities need to be consistent with the model of the strong interaction used in the equation of state for protoneutron star matter. Specifically, a particular choice of the particle-hole interaction should be consistent with the EOS employed to compute the composition of charge-neutral $\beta$-equilibrated matter [9]. In this paper we use the EOS based on the Skyrme effective interaction to compute $v_{e}$ and $\bar{v}_{e}$ charged-current opacities in the outer region of PNS called the neutrinosphere. We employ self-consistent RPA calculations, i.e., both the Hartree-Fock mean-field and the residual $p h$ interaction are derived from the same Skyrme potential. In this work we will consider five Skyrme parameterizations: KDE0v1, LNS, NRAPR, SKRA, SQMC700. It was shown in Ref. [10] that these Skyrme forces satisfy a series of experimentally extracted constraints for infinite nuclear matter properties.

The paper is organized as follows. In Sec. 2 we briefly present the Skyrme-RPA formalism to compute the Fermi and Gamow-Teller strength functions which determine the absorption cross section for $v_{e}$ and $\bar{v}_{e}$. Our results for the Fermi and Gamow-Teller strength functions as well as for (anti)neutrino opacities are presented in Sec. 3. Finally, we conclude in Sec. 4.

\section{Formalism}

The charged-current opacity for $v_{e}$ and $\bar{v}_{e}$ can be calculated by numerical integration of the absorption differential cross section per unit volume. In the nonrelativistic 
limit for nucleons, the double-differential cross-section for a (anti)neutrino with initial energy $E_{v}$ is given by [6]

$$
\begin{aligned}
& \frac{1}{V} \frac{d^{2} \sigma}{d \cos \theta d E_{e}}=\frac{G_{F}^{2} \cos ^{2} \theta_{C}}{2 \pi} p_{e} E_{e}\left(1-f_{e}\left(E_{e}\right)\right) \\
& \times\left[(1+\cos \theta) S_{0}^{( \pm)}(\omega, q)+g_{A}^{2}(3-\cos \theta) S_{1}^{( \pm)}(\omega, q)\right] .
\end{aligned}
$$

The energy transfer to the nuclear medium is $\omega=E_{v}-E_{e}$, while the momentum transfer is $q=|\vec{q}|=\left|\vec{p}_{v}-\vec{p}_{e}\right| . S_{0}^{( \pm)}$ and $S_{1}^{( \pm)}$are the strength functions (also called dynamical structure factors) associated with the Fermi and GamowTeller operators, $\vec{\tau}^{( \pm)}$and $\vec{\sigma} \vec{\tau}^{( \pm)}$, respectively. Note, that in Eq. (1) the plus sign refers to a neutrino absorption, while the minus sign refers to an antineutrino absorption. Thus, for (anti)neutrino absorption the cross section includes Fermi and Gamow-Teller strength functions for $n \rightarrow p$ $(p \rightarrow n)$ transitions. Finally, $1-f_{e}\left(E_{e}\right)$ is the Pauli blocking factor for the outgoing lepton. In the protoneutron star matter positrons are nondegenerate and their spectrum approaches the Boltzmann distribution. Therefore, we can neglect the final-state blocking for antineutrino absorption.

The strength functions $S_{0,1}^{( \pm)}$embody all spatial and temporal correlations between nucleons arising from strong interaction. At $T \neq 0$ they include summation of transition probabilities from thermally excited states $|i\rangle$ to final states $|f\rangle$ such that $\omega=E_{f}-E_{i}$ :

$$
S_{\alpha}^{( \pm)}(q, \omega)=\sum_{i, f} P_{i}\left|\left\langle f\left|\vec{Q}_{\alpha}^{( \pm)}\right| i\right\rangle\right|^{2} \delta\left(E_{f}-E_{i}-\omega\right),
$$

with $P_{i}=Z^{-1} \mathrm{e}^{-E_{i} / T}$ being the probability of finding the system in the state $|i\rangle$, and $Z=\sum_{i} e^{-E_{i} / T}$ is the partition function. The one-body transition operators $\vec{Q}_{\alpha}^{( \pm)}$are defined as $\vec{Q}_{0}^{( \pm)}=\sum_{j} e^{i \vec{q} \vec{r}_{j}} \vec{\tau}_{j}^{( \pm)}, \vec{Q}_{1}^{( \pm)}=\sum_{j} e^{i \vec{q} \vec{r}_{j}} \vec{\sigma}_{j} \vec{\tau}_{j}^{( \pm)}$, where the index $j$ stands for the nucleon. The strength functions obey the detailed balance theorem [11]

$$
S_{\alpha}^{( \pm)}(q, \omega)=e^{\left(\omega \pm \Delta \mu_{n p}\right) / T} S_{\alpha}^{(\mp)}(q,-\omega),
$$

where $\Delta \mu_{n p}=\mu_{n}-\mu_{p}$ is the difference between the neutron and proton chemical potentials, as well as they satisfy the non-energy weighted sum rule [11]

$$
\int_{-\infty}^{+\infty} d \omega\left[S_{\alpha}^{(+)}(q, \omega)-S_{\alpha}^{(-)}(q, \omega)\right]=\rho_{n}-\rho_{p},
$$

which depends only on the difference between neutron and proton densities.

We obtain the strength functions from the imaginary part of the response function $\Pi^{(\alpha)}(q, \omega)$ according to the fluctuation-dissipation theorem

$$
S_{\alpha}^{( \pm)}(q, \omega)=-\frac{1}{\pi} \frac{\mathfrak{I} m \Pi_{\alpha}^{( \pm)}(q, \omega)}{1-\mathrm{e}^{-\left(\omega \pm \Delta \mu_{n p}\right)}} .
$$

In its turn $\Pi_{\alpha}^{( \pm)}$is related to the particle-hole propagator (or the retarded $p h$ Green's function)

$$
\Pi_{\alpha}^{( \pm)}(q, \omega)=2 \int \frac{d^{3} \vec{k}}{(2 \pi)^{3}} G_{\alpha}^{( \pm)}(k, q, \omega) .
$$

Here and below, for the momentum averages we adopt the notation

$$
\int \frac{d^{3} \vec{k}}{(2 \pi)^{3}} f(\vec{k}) G_{\alpha}^{( \pm)}(k, q, \omega) \equiv\left\langle f G_{\alpha}^{( \pm)}\right\rangle .
$$

With this notation $\Pi_{\alpha}^{( \pm)}(q, \omega)=2\left\langle G_{\alpha}^{( \pm)}\right\rangle$.

Neglecting long-range $p h$ correlations $G_{\alpha}^{( \pm)}=G_{H F}^{( \pm)}$, and the Hartree-Fock particle-hole propagator is given by

$$
G_{H F}^{( \pm)}(k, q, \omega)=\frac{f_{n(p)}(\vec{k})-f_{p(n)}(\vec{k}+\vec{q})}{\omega+E_{n(p)}(\vec{k})-E_{p(n)}(\vec{k}+\vec{q})+i \eta} .
$$

Here $f_{\tau}(\vec{k})=\left[1+\mathrm{e}^{\left(E_{\tau}(\vec{k})-\mu_{\tau}\right) / T}\right]^{-1}$ is the Fermi-Dirac distribution for nucleons and $E_{\tau}(\vec{k})$ is the single particle energy given by

$$
E_{\tau}(\vec{k})=\frac{\vec{k}^{2}}{2 m_{\tau}}+U_{\tau},
$$

where $m_{\tau}$ is the effective mass and $U_{\tau}$ is the HartreeFock potential obtained with the Skyrme interaction. Using the detailed balance (3) we can write the Hartree-Fock strength function for $n \rightarrow p(p \rightarrow n)$ transitions as

$$
\begin{aligned}
S_{H F}^{( \pm)}(q, \omega)=2 \int & \frac{d^{3} \vec{k}}{(2 \pi)^{3}} \delta\left(E_{p(n)}-E_{n(p)}-\omega\right) \\
& \times f_{n(p)}(\vec{k})\left(1-f_{p(n)}(\vec{k}+\vec{q})\right) .
\end{aligned}
$$

Assuming $U_{\tau}=0$ and considering the bare nucleon masses we get the strength function for a noninteracting Fermi gas.

To go beyond the HF approximation we take into the residual proton-neutron interaction by resumming a class of so-called ring diagrams and obtain the well known RPA [12]. The RPA correlated ph propagator is the solution of the Bethe-Salpeter integral equation

$$
\begin{aligned}
& G_{\alpha}^{( \pm)}\left(k_{1}, q, \omega\right)=G_{H F}^{( \pm)}\left(k_{1}, q, \omega\right)+G_{H F}^{( \pm)}\left(k_{1}, q, \omega\right) \\
& \times \sum_{\alpha^{\prime}} \int \frac{d^{3} \overrightarrow{k_{2}}}{(2 \pi)^{3}} V_{p h}^{\left(\alpha, \alpha^{\prime}\right)}\left(\vec{q}, \vec{k}_{1}, \vec{k}_{2}\right) G_{\alpha^{\prime}}^{( \pm)}\left(k_{2}, q, \omega\right),
\end{aligned}
$$

where $V_{p h}^{\left(\alpha, \alpha^{\prime}\right)}$ is the residual interaction matrix element. For the Skyrme effective interaction containing a zerorange spin-orbit term this proton-neutron matrix element takes the form [13]

$$
\begin{aligned}
& V_{p h}^{\left(S M ; S^{\prime} M^{\prime}\right)}\left(\vec{q}, \vec{k}_{1}, \vec{k}_{2}\right) \equiv \\
& \left\langle\vec{q}+\vec{k}_{1}, \vec{k}_{1}^{-1} ; S M\left|V_{p h}\right| \vec{q}+\vec{k}_{2}, \vec{k}_{2}^{-1} ; S^{\prime} M^{\prime}\right\rangle= \\
& \quad \delta_{S S^{\prime}} \delta_{M M^{\prime}}\left(W_{1}^{(S)}+W_{2}^{(S)} \vec{k}_{12}^{2}\right) \\
& +q W_{S O}^{(1)}\left(\delta_{S^{\prime} 0} \delta_{S 1} M\left(k_{12}\right)_{-M}^{(1)}+\delta_{S^{\prime} 1} \delta_{S 0} M^{\prime}\left(k_{12}\right)_{M^{\prime}}^{(1)}\right),
\end{aligned}
$$

where $\vec{k}_{12}=\vec{k}_{1}-\vec{k}_{2}$ is the relative hole momentum, while the rank-1 tensor $\left(k_{12}\right)_{M}^{(1)}$ is defined in [13]. The coefficients $W_{1,2}^{(S)}$ are the combinations of the Skyrme parameters $\left(x_{i}, t_{i}\right)$, the transferred momentum $q$ and the total density $\rho=\rho_{n}+\rho_{p}$. Their detailed expressions are given in [11]. It is important to note that the spin-orbit term introduces a coupling between $S=0$ and $S=1$ spin channels and removes the degeneracy on the spin projection $M$. 
Following the method suggested by Margueron et al. [14], a closed algebraic system for $\left\langle G_{\alpha}^{( \pm)}\right\rangle$is obtained by multiplying the Bethe-Salpeter equation (11) with the functions $k_{1}^{2}, k_{1} Y_{1 \mu}$ and integrating over the momentum. Finally, the system of algebraic equations can be written in a compact form for each channel $(\alpha=S, M)$ as

$$
\begin{gathered}
\left(1-\widetilde{W}_{1}^{(\alpha)} \beta_{0}^{( \pm)}-W_{2}^{(\alpha)} q^{2} \beta_{2}^{( \pm)}\right)\left\langle G_{\alpha}^{( \pm)}\right\rangle \\
-W_{2}^{(\alpha)} \beta_{0}^{( \pm)}\left\langle k^{2} G_{\alpha}^{( \pm)}\right\rangle+2 W_{2}^{(\alpha)} q \beta_{1}^{( \pm)} \\
\times \sqrt{\frac{4 \pi}{3}}\left\langle k Y_{10} G_{\alpha}^{( \pm)}\right\rangle=\beta_{0}^{( \pm)}, \\
-\left(\widetilde{W}_{1}^{(\alpha)} q^{2} \beta_{2}^{( \pm)}+W_{2}^{(\alpha)} q^{4} \beta_{5}^{( \pm)}\right)\left\langle G_{\alpha}^{( \pm)}\right\rangle \\
+\left(1-W_{2}^{(\alpha)} q^{2} \beta_{2}^{( \pm)}\right)\left\langle k^{2} G_{\alpha}^{( \pm)}\right\rangle+2 W_{2}^{(\alpha)} q^{3} \beta_{4}^{( \pm)} \\
\times \sqrt{\frac{4 \pi}{3}}\left\langle k Y_{10} G_{\alpha}^{( \pm)}\right\rangle=q^{2} \beta_{2}^{( \pm)}, \\
-\left(\widetilde{W}_{1}^{(\alpha)} q \beta_{1}^{( \pm)}+W_{2}^{(\alpha)} q^{3} \beta_{4}^{( \pm)}\right)\left\langle G_{\alpha}^{( \pm)}\right\rangle \\
-W_{2}^{(\alpha)} q \beta_{1}^{( \pm)}\left\langle k^{2} G_{\alpha}^{( \pm)}\right\rangle+\left(1+2 W_{2}^{(\alpha)} q^{2} \beta_{3}^{( \pm)}\right) \\
\times \sqrt{\frac{4 \pi}{3}}\left\langle k Y_{10} G_{\alpha}^{( \pm)}\right\rangle=q \beta_{1}^{( \pm)} .
\end{gathered}
$$

The parameter $\widetilde{W}_{1}^{(\alpha)}$ describes the coupling between spin channels induced by the spin-orbit interaction

$$
\widetilde{W}_{1}^{(\alpha)}=W_{1}^{(\alpha)}+C^{(\alpha)} \frac{W_{S O}^{2} q^{4}\left(\beta_{2}^{( \pm)}-\beta_{3}^{( \pm)}\right)}{1+W_{2}^{\left(\alpha^{\prime}\right)} q^{2}\left(\beta_{2}^{( \pm)}-\beta_{3}^{( \pm)}\right)} .
$$

Here $\alpha^{\prime}$ is defined with respect to $\alpha$ as $S^{\prime}=1-S$, while $C^{(\alpha)}=1$ if $S=0$ and $C^{(\alpha)}=\frac{1}{2} M^{2}$ if $S=1$. The six functions $\beta_{l}^{( \pm)}(q, \omega),(l=0,5)$ include the momentum averages of the HF propagator $G_{H F}^{( \pm)}$and their explicit expressions are given [11]. If we replace $\widetilde{W}_{1}^{(\alpha)}$ in Eqs. (12-14) by $W_{1}^{(\alpha)}$ we obtain the results of Ref. [11], related to the central part of the interaction, as it should be. We also would like to note that for ambient conditions (density and temperature) considered in the present work, the effect of the spin-orbit interaction is negligible and for Gamow-Teller strength function we have $S_{S=1, M=0}^{( \pm)} \approx S_{S=1, M=1}^{( \pm)} \equiv S_{S=1}^{( \pm)}$.

\section{Results}

To illustrate the general features of the strength functions we have chosen the LNS Skyrme parametrization. The temperature evolution of Fermi and GT strength functions is shown in Fig. 1 for both $n \rightarrow p$ and $p \rightarrow n$ transition at a small momentum transfer $q=0.2 \mathrm{fm}^{-1}$. We have performed calculations at density $\rho=0.02 \mathrm{fm}^{-3}$ for a proton fraction $Y_{p}=\rho_{p} / \rho=0.1$. As a reference, the HF and noninteracting Fermi gas (FG) strength functions - which are independent of the spin channel - are also shown.

Comparing the noninteracting $n \rightarrow p$ FG strength functions with the HF ones we find that they have the same shape, but the interaction shifts the HF strength function to larger negative energy transfer. It means that the energy of
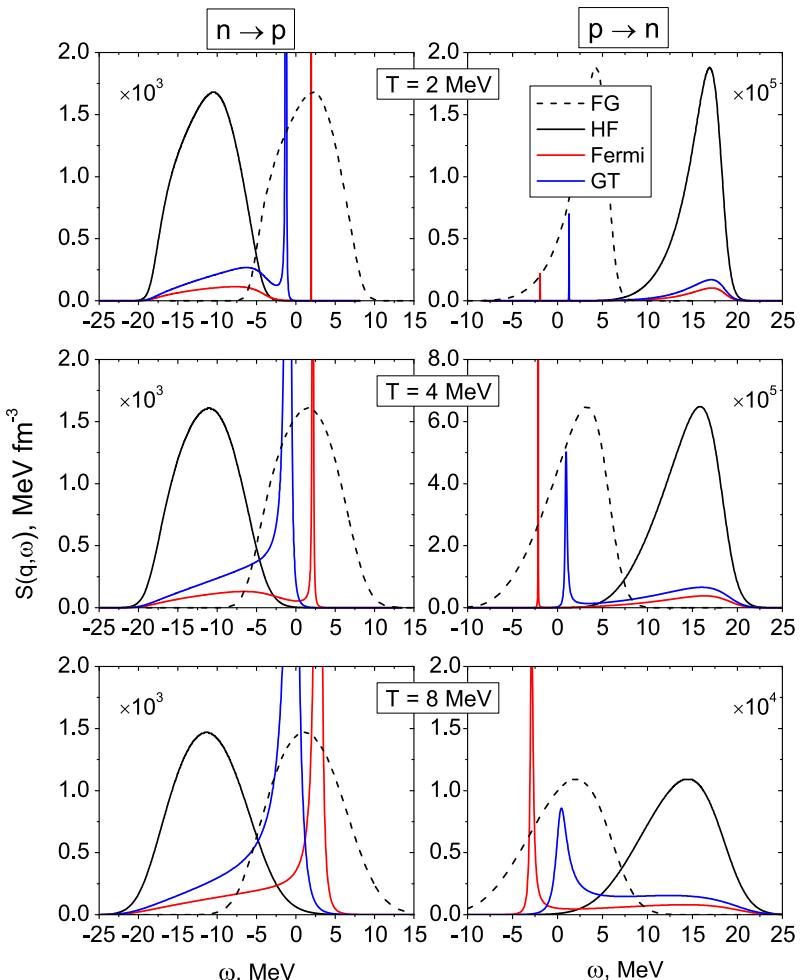

Figure 1. (Color online) The finite temperature $(T=2,4,8 \mathrm{MeV})$ $\mathrm{HF}$ and RPA strength functions at $q=0.2 \mathrm{fm}^{-3}$ as functions of energy $\omega$ for $n \rightarrow p$ (left panels) and $p \rightarrow n$ (right panels) transitions. The dashed curves give the strength function for the noninteracting Fermi gas (FG). Note, that the strength functions are scaled by the factor shown inside each panel.

the nuclear system is decreased for $n \rightarrow p$ transitions. In contrast, the interaction pushes the HF $p \rightarrow n$ strength function to higher energy transfer, thereby increasing the energy of the nuclear system. This is understood as follows. The dispersion relation for the nucleon is given by Eq. (9) and the energy shift $\Delta U_{n p}=U_{n}-U_{p}$ is closely related to the symmetry energy, which describes how the energy of nuclear matter changes as one moves away from the equal number of protons and neutrons. For the LNS Skyrme interaction the potential difference at considered neutron-rich conditions is $\Delta U_{n p} \approx 12 \mathrm{MeV}$. Thus the proton is more strongly bound than the neutron and the difference in potentials serves as a threshold for the $p \rightarrow n$ reactions.

Allowing for repulsive $p h$ interaction at low temperatures significantly suppresses the continuum part of the HF strength functions and gives rise to well-defined Fermi and Gamow-Teller collective modes either at positive or negative energy above the particle-hole band. Moreover, due to the detailed balance (3), each collective mode peak in the $n \rightarrow p$ strength function has its image in the $p \rightarrow n$ strength function at $-\omega$. One important point is that these $p \rightarrow n$ collective modes appear only due to thermal effects and they are located below the particle-hole band. The centroid of collective excitations is almost stable against thermal effects, while their width grows with increasing temperature due to Landau damping. At high tempera- 

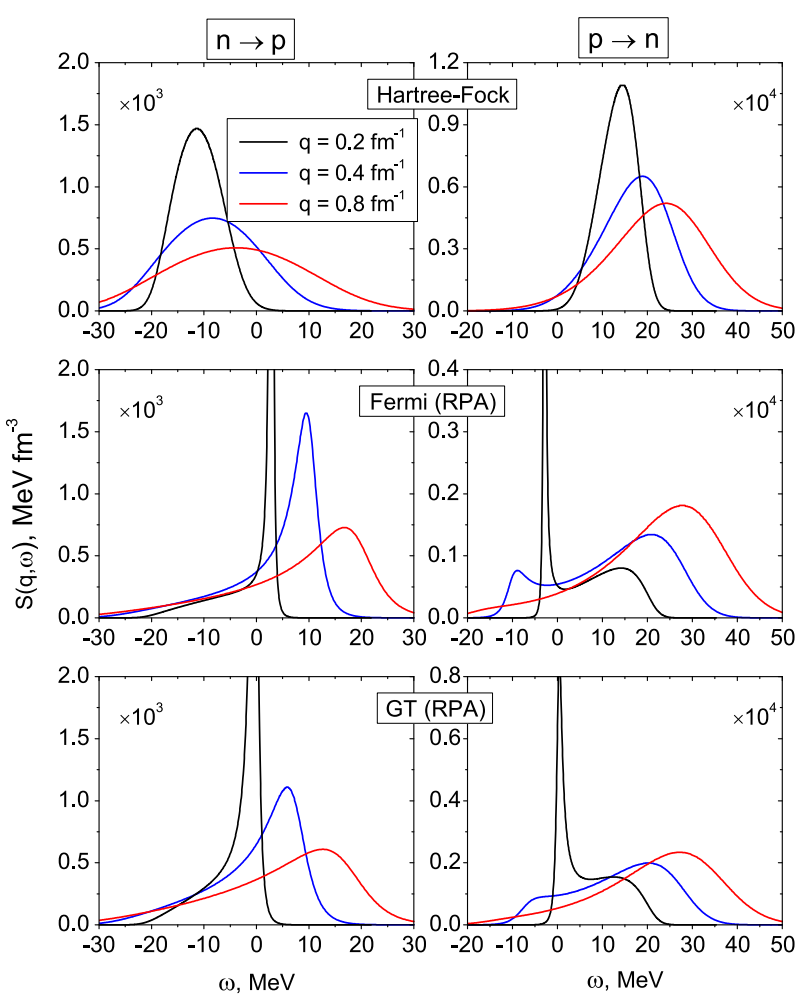

Figure 2. (Color online) Finite temperature $(T=8 \mathrm{MeV})$ strength functions calculated with the LNS Skyrme potential at three values of momentum transfer. The density and the proton fraction are the same as in Fig. 1

tures collective modes become absorbed into thermally extended particle-hole band.

Figure 2 shows a typical modification of the Fermi and Gamow-Teller strength functions, $S_{0}^{( \pm)}$and $S_{1}^{( \pm)}$, with increasing momentum transfer. The HF strength functions are shown in the same figure for reference. As we have mentioned before and as it follows from Eq. (10), the evolution of the HF strength functions for $n \rightarrow p(p \rightarrow n)$ transitions follows that of the noninteracting Fermi gas, but shifted by $\Delta U_{n p} \approx 12 \mathrm{MeV}$ to lower (higher) energies. In particular, we can observe the enlarging the particlehole band with increasing momentum transfer $q$ following the overall reduction of the strength. The sum rule (4) requires that for neutron-rich conditions the momentum increase has a smaller effect on the total $n \rightarrow p$ strength than on that for the $p \rightarrow n$ transitions.

The middle and lower panels of Fig. 2 show that the effects of increasing momentum are remarkably similar for the Fermi and Gamow-Teller strength functions. At low momentum transfer the $n \rightarrow p(p \rightarrow n)$ RPA strength functions in both the $S=0$ and $S=1$ channels have relatively narrow peaks above (below) the maximum of the HF strength. When $q$ increases, the peaks become broader and finally the RPA strength evolves to a structureless shape. Furthermore, although at large $q$ the $p h$ correlations have a relatively smaller effect than at small $q$, the $n \rightarrow p$ RPA strength functions remain shifted to larger energies with respect to the HF strength. In contrast, because of diminishing contribution from thermally excited collective modes, the maximums of the $p \rightarrow n$ Fermi and GamowTeller strength functions move from low energies to higher ones and finally they appear above the HF maximum.

The differential absorption cross sections for neutrinos and antineutrinos as a function of the outgoing lepton energy are shown in Fig. 3. We consider incoming neutrino energies $E_{v, \bar{v}}=10,20,50$ and $100 \mathrm{MeV}$ and the conditions of the medium are $\rho=0.02 \mathrm{fm}^{-3}, T=8 \mathrm{MeV}$. The proton fraction and the electron chemical potential are $Y_{p}=0.029$ and $\mu_{e}=47.05 \mathrm{MeV}$, respectively, which correspond to beta-equilibrium (i.e., $\mu_{e}=\mu_{n}-\mu_{p}$ ) for the given temperature, density and the assumed nuclear Skyrme interaction LNS. The mean-field potential difference is $\Delta U_{n p}=14.66 \mathrm{MeV}$.

It is seen from the figure, the peak of the differential cross section is shifted by about $\Delta U_{n p}$ to higher (lower) energies for (anti)neutrino absorption with respect to the noninteracting Fermi gas model and, hence, the meanfield potential contribution increases (reduces) the energy of the emitted electron (positrons). This observation is in line with the elastic approximation [1] where the energy of the outgoing electron (positron) is $E_{e}=E_{v}+\Delta U_{n p}$ $\left(E_{e^{+}}=E_{\bar{v}}-\Delta U_{n p}\right)$. For low energy neutrinos, $E_{v}<\mu_{e}$, the additional energy $\Delta U_{n p}$ is not enough to overcome the Pauli blocking for the outgoing electron. However, this energy is sufficient to put the outgoing electron in a less blocked portion of phase space, thereby leading to an exponential enhancement of the cross section. For highenergy neutrinos, $E_{v}>\mu_{e}$, the cross section is approximately proportional to the phase space factor $p_{e} E_{e}$ and the effects of the mean field contribution diminish with increasing $E_{v}$.

For the antineutrino HF cross section the important difference from the neutrino case comes from the fact that the reaction threshold is increased by $\Delta U_{n p}$. The increased reaction threshold leads to a significant suppression of the HF cross section for low-energy antineutrinos. For $E_{\bar{\gamma}}<\Delta U_{n p}$ antineutrinos the HF cross section differs from zero due to thermal effects which smear the proton and neutron Fermi surfaces. As was stated above, we can neglect the Pauli blocking for the outgoing positron and for $E_{\bar{v}}>\Delta U_{n p}$ antineutrinos the HF cross section is suppressed solely by the reduced available phase space.

Let us now consider the role of RPA correlations on the differential cross sections. It is seen from the upper panels of Fig. 3 that RPA correlations suppress the neutrino absorption cross section. The degree of suppression depends on neutrino energy and it is most significant for low-energy neutrinos when the transferred momentum is also low and the RPA strength functions are dominated by the resonance contributions (see Fig. 2). The GamowTeller and Fermi resonances are clearly visible in the differential cross section for low-energy neutrinos, however their contribution is strongly suppressed by the phasespace blocking for outgoing electrons. With increasing neutrino energy, the contribution of collective states to the $v_{e}$ cross section decreases and the RPA corrections become smaller. Concerning $\bar{v}_{e}$ absorption, for low-energy antineutrinos the HF cross section is almost zero due to reaction threshold. However, RPA correlations and ther- 

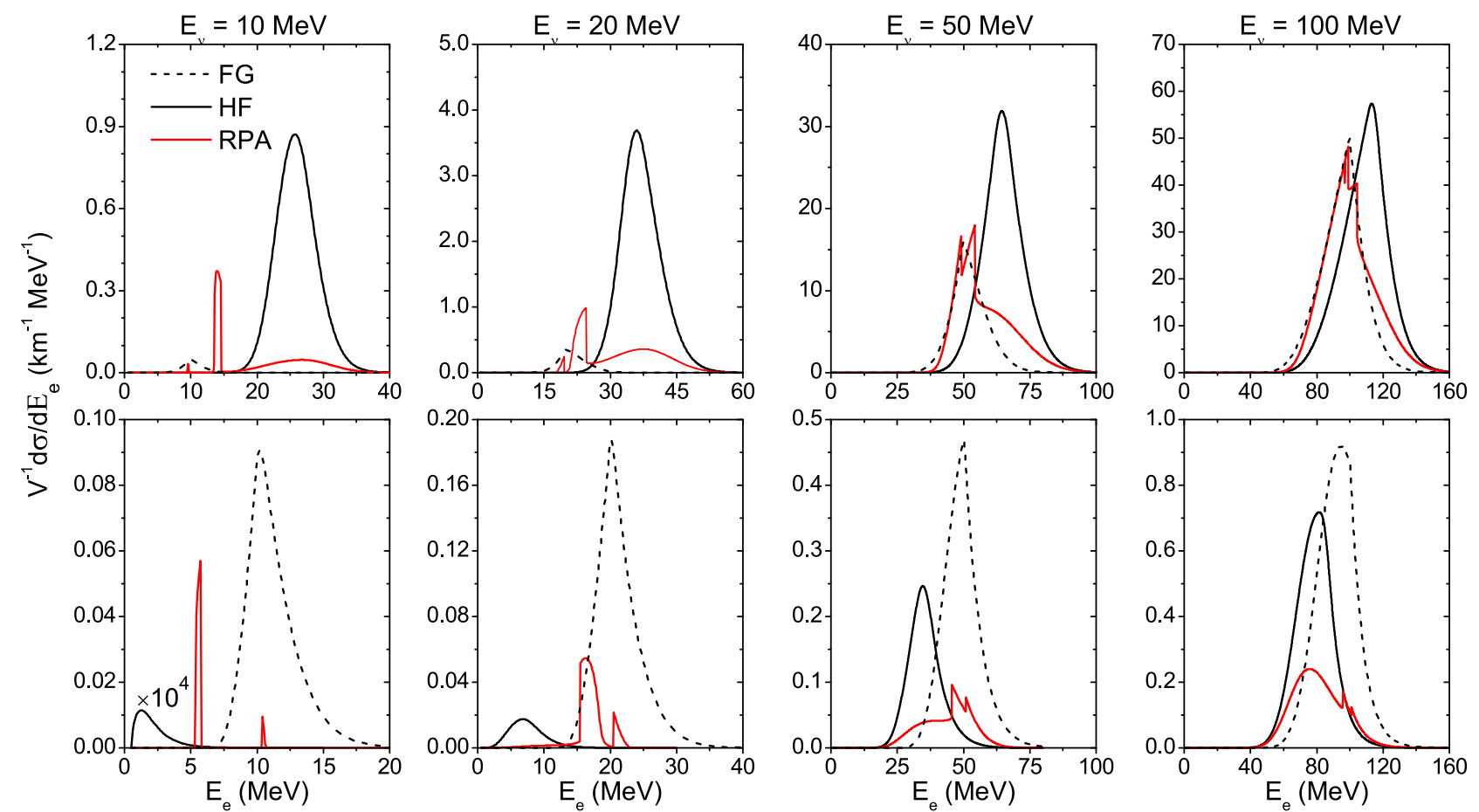

Figure 3. (Color online) Differential cross sections for neutrino (upper panels) and antineutrino (lower panels) absorption, evaluated at beta-equilibrium conditions $\rho=0.02 \mathrm{fm}^{-3}, T=8 \mathrm{MeV}$, and $Y_{p}=0.029$. The Skyrme force LNS is used in the HF and RPA calculations. In each panel the dashed line shows the noninteracting Fermi gas cross sections. For antineutrinos with $E_{\bar{v}_{e}}=10 \mathrm{MeV}$ the HF cross section is scaled by the factor $10^{4}$.

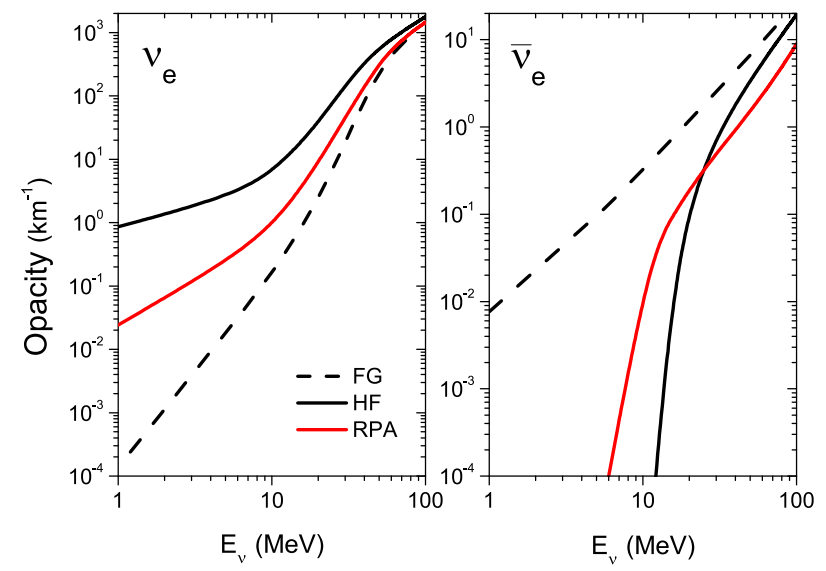

Figure 4. (Color online) Neutrino (left panel) and antineutrino (right panel) opacities, evaluated at beta-equilibrium conditions $\rho=0.02 \mathrm{fm}^{-3}, T=8 \mathrm{MeV}$, and $Y_{p}=0.029$ with the Skyrme force LNS.

mal effects give rise to collective peaks which decrease the reaction threshold and enhance the cross section significantly. The contribution of collective states diminishes with increasing antineutrino energy and the RPA corrections tend to reduce the cross section as in the $v_{e}$ case.

Figure 4 shows neutrino and antineutrino opacities evaluated at the same conditions considered in Fig. 3. The results shown in Fig. 4 follow the trends expected from the results for the differential cross sections. Namely, due to presence of the mean-field potentials the opacity for lowenergy neutrinos increases with respect to the noninteracting case, but at larger neutrino energies the mean-field contribution becomes less important and the opacities computed with and without mean-field potentials approach each other asymptotically. In contrast, the antineutrino HF opacity is reduced relative to the Fermi gas result and the reaction threshold manifests itself at $\Delta U_{n p}$. For incoming (anti)neutrino energy $E_{v}=24 \mathrm{MeV}$ which is the mean thermal energy $E_{v} \sim 3 T$ at temperature $T=8 \mathrm{MeV}$ the HF neutrino opacity is enhanced by about an order of magnitude compared to the noninteracting Fermi gas, while the antineutrino opacity is suppressed by almost a factor of 5. While mean-field effect tends to increase the neutrino opacity, RPA correlations decrease it. For $E_{v}=24 \mathrm{MeV}$ neutrinos, the RPA opacity is suppressed by almost a factor of 4 relative to the HF one. For antineutrino absorption, the inclusion of RPA correlations decreases the reaction threshold and increases opacity for low-energy neutrinos. However, at higher neutrino energies RPA correlations reduces the opacity.

We now compare the opacities evaluated with different Skyrme forces mentioned in the Introduction. Results for the parametrizations KDE0v1, LNS, NRAPR, SKRPA, and SQMC700 are shown in Fig. 5. The RPA opacities do not differ significantly between the last four Skyrme parametrizations. In contrast, the HF calculations based on the KDE0v1 force predict a mean-field potential difference $\Delta U_{n p}=20.7 \mathrm{MeV}$ which is substantially larger than that for other four parametrizations. As a result, low- 


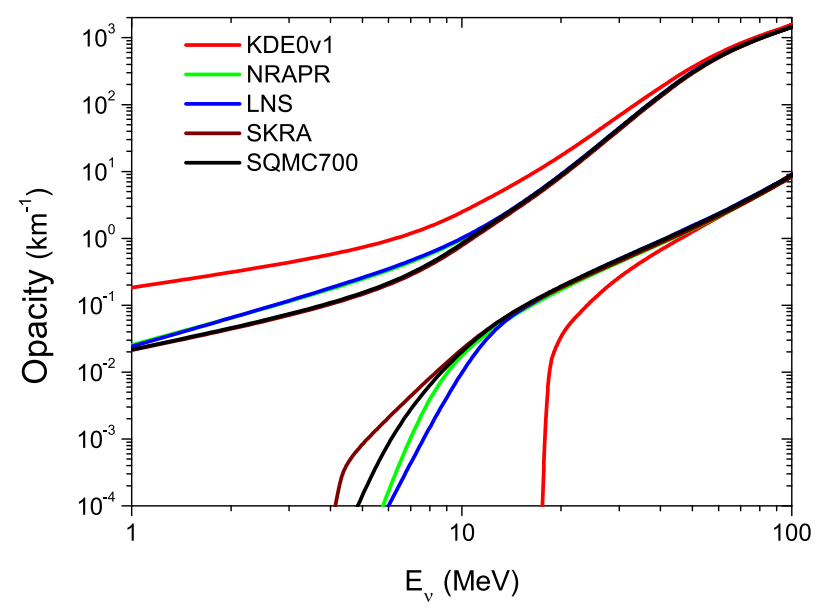

Figure 5. (Color online) Opacity for neutrino and antineutrino, evaluated at conditions $\rho=0.02 \mathrm{fm}^{-3}, T=8 \mathrm{MeV}$, and $Y_{p}=$ 0.029 with the different Skyrme parameterizations.

energy neutrino (antineutrino) opacities evaluated with the KDE0v1 force turn out to be well above (below) those expected from other Skyrme forces.

\section{Conclusions}

In this paper, we have studied the combined effects of the mean-field potential difference and RPA correlations on the $v_{e}$ and $\bar{v}_{e}$ opacity in the matter of protoneutron stars. By solving the Bethe-Salpeter equation with the residual particle-hole interaction derived from a Skyrme potential, we estimated the Fermi and Gamow-Teller strength functions which are directly related to the (anti)neutrino absorption cross section. Strength functions are analyzed for varying conditions of momentum transfer and temperature for a representative Skyrme potential LNS. It is shown that both mean-field effects and RPA correlations play a significant role in the strength function redistribution relative to the noninteracting Fermi gas. In agreement with the previous studies [5, 6], the mean-field corrections enhance the neutrino opacity and suppress the antineutrino opacity. Our calculations, however, indicate that RPA correlations may significantly suppress the HF neutrino opacity at thermal neutrino energies in agreement with previ- ous studies [9, 15]. For antineutrino absorption the relative contribution of RPA corrections is energy dependent: for near threshold antineutrinos they enhance the opacity, while for higher energies they act in the opposite direction. The joint effect of mean-field energy shift and long-range RPA correlations make the protoneutron star matter more opaque (transparent) for (anti)neutrino radiation as compared to the noninteracting Fermi gas model.

This work was supported by the Heisenberg-Landau Program. G.M.-P. is partly supported by the Deutsche Forschungsgemeinschaft through contract SFB 1245.

\section{References}

[1] S. Reddy, M. Prakash, J.M. Lattimer, Phys. Rev. D 58, 013009 (1998)

[2] A. Burrows, S. Reddy, T.A. Thompson, Nucl. Phys. A 777, 356 (2006)

[3] H. Bethe, J. Wilson, Astrophys. J. 295, 14 (1985)

[4] H.T. Janka, Annual Review of Nuclear and Particle Science 62, 407 (2012)

[5] G. Martínez-Pinedo, T. Fischer, A. Lohs, L. Huther, Phys. Rev. Lett. 109, 251104 (2012)

[6] L.F. Roberts, S. Reddy, G. Shen, Phys. Rev. C 86, 065803 (2012)

[7] R.F. Sawyer, Phys. Rev. D 11, 2740 (1975)

[8] R.F. Sawyer, Phys. Rev. C 40, 865 (1989)

[9] S. Reddy, M. Prakash, J.M. Lattimer, J.A. Pons, Phys. Rev. C 59, 2888 (1999)

[10] M. Dutra, O. Lourenço, J.S. Sá Martins, A. Delfino, J.R. Stone, P.D. Stevenson, Phys. Rev. C 85, 035201 (2012)

[11] E. Hernández, J. Navarro, A. Polls, Nucl. Phys. A 658, 327 (1999)

[12] A.L. Fetter, J.D. Walecka, Quantum Theory of ManyParticle Systems (McGraw-Hill, Boston, 1971)

[13] D. Davesne, A. Pastore, J. Navarro, Phys. Rev. C 89, 044302 (2014)

[14] J. Margueron, N.V. Giai, J. Navarro, Phys. Rev. C 74, 015805 (2006)

[15] A. Burrows, R.F. Sawyer, Phys. Rev. C 59, 510 (1999) 